\section{TRANSIENT RESPONSE OF A CLASS OF NONLINEAR SYSTEMS}

An algorithm is given to be used in conjunction with the parameter-plane method and the describing-function method for rapid calculation of transient oscillations in the design of a class of nonlinear systems.

This letter gives a straightforward algorithm for plotting the zero-input response of a class of nonlinear systems with arbitrary initial conditions.

The classes of nonlinear systems considered are those for which the stability of self-excited oscillations are determined by the nonlinear differential equation

$$
C(s) x+B(s) F(x)=0 \text {. }
$$

where $s=d / d t, C(s)$ and $B(s)$ are polynomials in $s$ with the degree of $C(s)$ being higher than the degree of $B(s)$, and the function $F(x)$ represents the nonlinearity. It will be assumed that the relative stability of nonlinear control systems described by eqn. 1 may be studied in the parameter plane using the approach of Krylov and Bogoliubov and the describing-function method. ${ }^{1-3}$ On the basis of this approach, the nonlinear function $F(x)$ is 'linearised' as

$$
F(x)=N_{1}(A) x
$$

where

$$
N_{1}(A)=\frac{1}{\pi A} \int_{0}^{2 \pi} F(A \sin \phi) \sin \phi d \phi
$$

The linearised differential equation corresponding to eqn. 1 is thus

$$
\left\{C(s)+B(s) N_{1}(A)\right\} x=0
$$

The advantage of using the parameter-plane approach in conjunction with the describing-function method is that insight is given as to the effects of the various parameters on transient behaviour in the preliminary stages of design. ${ }^{3}$ Also, any system adjustable parameters may be selected, when possible, so that the system equation (eqn. 4) may be approximated to by an equivalent second-order nonlinear system

$$
\left[s^{2}-2 \bar{\sigma}(t) s+\{\bar{\sigma}(t)\}^{2}+\{\bar{\omega}(t)\}^{2}\right] x-0
$$

which has the desired transient characteristic. The values of $\widetilde{\sigma}(t)$ and $\bar{\omega}(t)$ are determined from $A(t)$ and the functions $\bar{\sigma}(A)$ and $\bar{\omega}(A)$. The functions $\bar{\sigma}(A)$ and $\bar{\omega}(A)$ are determined using the nonlinearity characteristics $N_{1}(A)$, together with the parameter-plane constant $\sigma$ and $\omega$ contours or the constant $\zeta$ and $\omega_{n}$ contours, where $\sigma=\omega_{n} \zeta$ and $\omega=\omega_{n} \sqrt{ }\left(1-\zeta^{2}\right)^{2}$

It is the purpose of this letter to develop a straightforward algorithm for calculating $A(t)$, and thus the solution $x(t)$ of eqn. 5, for any specified initial conditions given $\bar{\sigma}(A)$ and $\bar{\omega}(A)$. The solution $x(t)$ may then be used as an approximation to the solution of the corresponding equation (eqn. 4). The developments are extensions of the methods of Grensted. ${ }^{4}$

Solutions of eqn. 5 are assumed to have the form

$$
x(t)=A(t) \sin \phi(t)
$$

where

$$
\frac{d A}{d t}=\sigma_{1} A \text { and } \frac{d \phi}{d t}=\omega_{1}
$$

i.e. solutions of eqn. 5 are given by

$$
x(t)=A_{0} \exp \left(\int_{t_{0}}^{t} \sigma_{1} d t\right) \sin \left(\int_{0}^{t} \omega_{1} d t+\phi_{0}\right) .
$$

Substituting eqn. 8 into eqn. 5 results in two equations valid for all $A$, i.e.

$$
\begin{aligned}
& \dot{\sigma}_{1}+\sigma_{1}^{2}-2 \bar{\sigma} \sigma_{1}-\omega_{1}^{2}+\bar{\sigma}^{2}+\bar{\omega}^{2}=0 \quad . \quad \text {. (9a) } \\
& 2 \sigma_{1} \omega_{1}+\dot{\omega}_{1}-2 \bar{\sigma} \omega_{1}=0 \quad . \quad(9 b)
\end{aligned}
$$

If the assumption is made that $\dot{\bar{\sigma}}=\dot{\bar{\omega}}=0$, eqns. $9 a$ and $b$ yield $\sigma_{1}=\bar{\sigma}, \omega_{1}=\bar{\omega}$, as in the Krylov-Bogoliubov analysis. By not neglecting the $\dot{\bar{\sigma}}$ and $\dot{\bar{\omega}}$ terms of eqns. $9 a$ and $b$, improved results are obtained. The equations may be rewritten neglecting second-order terms, giving $\sigma_{1}$ and $\omega_{1}$ in terms of $\bar{\sigma}, \bar{\omega}, \dot{\bar{\sigma}}$ and $\dot{\bar{\omega}}$, i.e.

$$
\bar{\sigma}_{1}=\bar{\sigma}-\frac{\bar{\omega} \dot{\bar{\omega}}}{2\left(\dot{\bar{\sigma}}+\bar{\omega}^{2}\right)} \omega_{1}^{2}=\sigma+\omega^{2}
$$

In order to calculate the constants $A_{0}$ and $\phi_{0}$ of eqn. 8 , consider its time derivative; i.e.

$$
\begin{array}{r}
\dot{x}(t)=A_{0} \exp \left(\int_{0}^{t} \sigma_{1} d t\right)\left\{\sigma_{1} \sin \left(\int_{t_{0}}^{t} \omega_{1} d t+\phi_{0}\right)\right. \\
\left.+\omega_{1} \cos \left(\int_{t_{0}}^{t} \omega_{1} d t+\phi_{0}\right)\right\}
\end{array}
$$

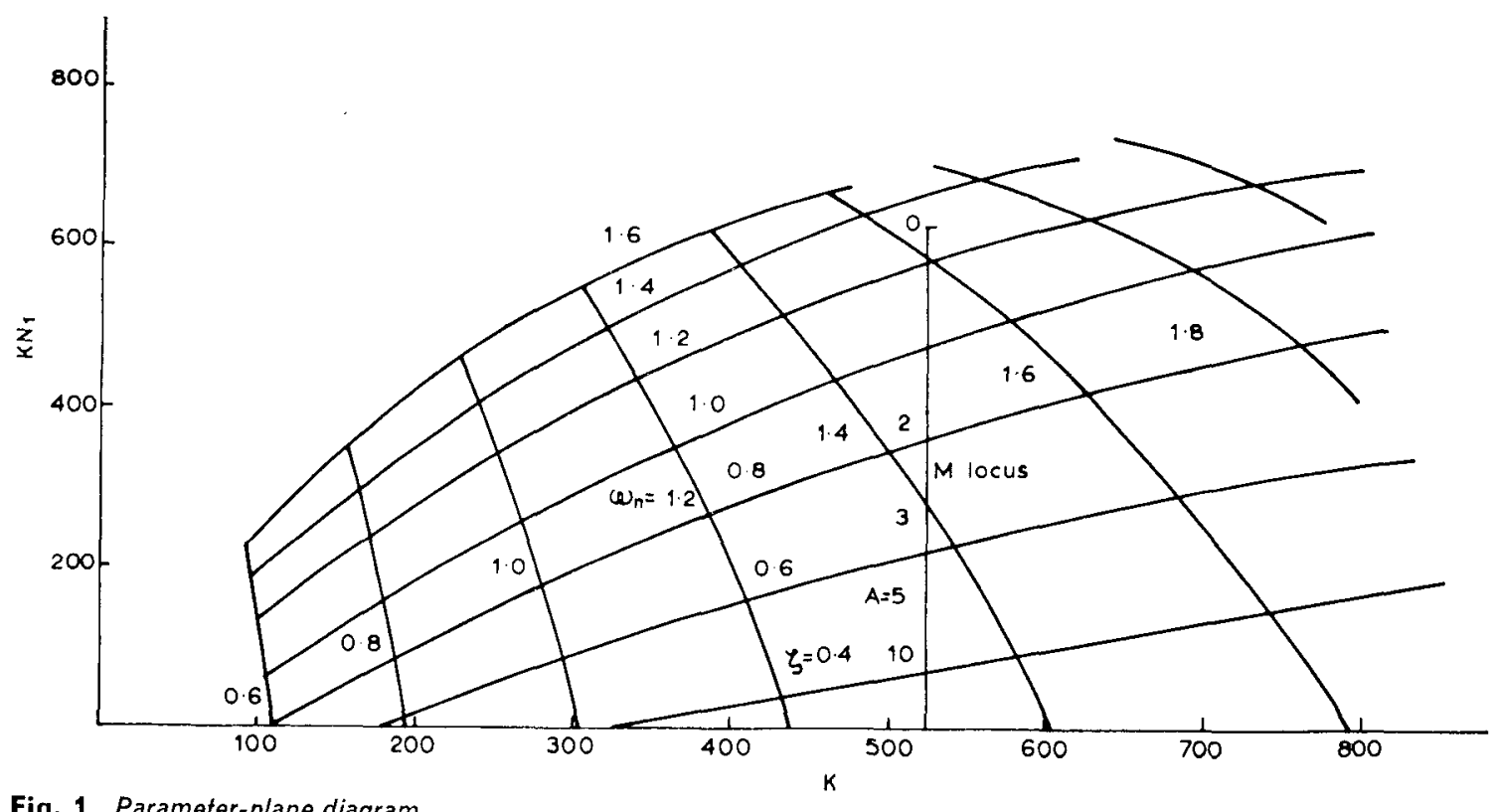

Fig. 1 Parameter-plane diagram 


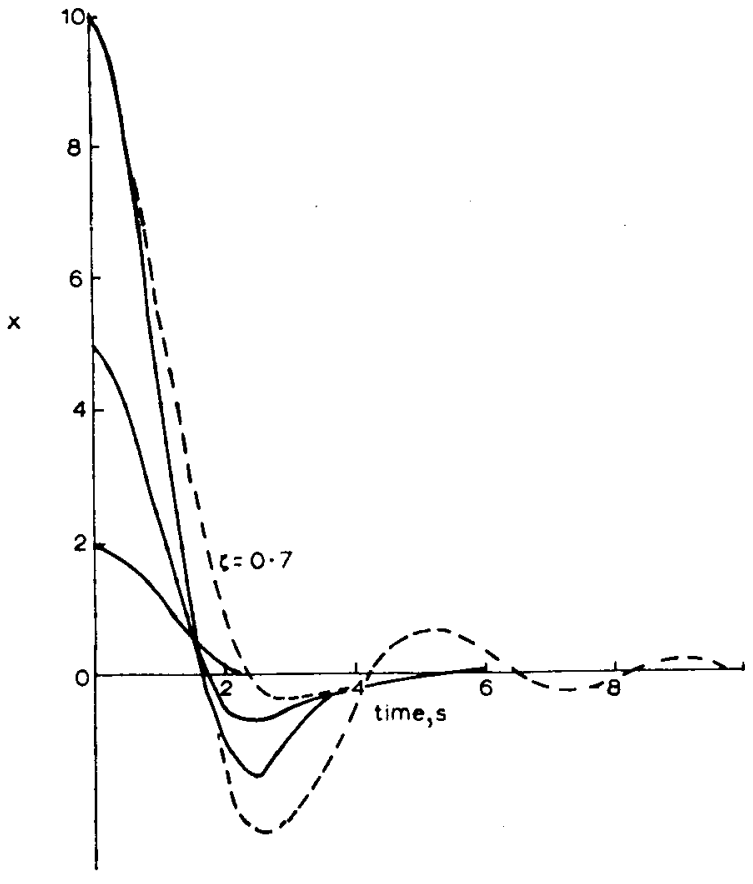

Fig. 2 System response

Assuming that the boundary conditions of eqn. 5 are given as $x\left(t_{0}\right)=x_{0}$ and $\dot{x}\left(t_{0}\right)=0$, eqns. 8 and 11 give $\phi_{0}$ and $A_{0}$ as

$$
\phi_{0}=\tan ^{-1} \frac{\omega_{1}\left(t_{0}\right)}{\sigma_{1}\left(t_{0}\right)} \quad A_{0}=\frac{x_{0}}{\sin \phi_{0}} .
$$

Thus $\phi_{0}$ and $A_{0}$ may be calculated from eqns. 12 and 10 using estimations for $\dot{\bar{\omega}}$ and $\dot{\bar{\sigma}}$ (perhaps just taken as zero), and the transient oscillations may be determined from eqn. 8 using the following algorithm:

(a) Read in $\bar{\sigma}(A), \bar{\omega}(A)$ curves and initial values of $\omega_{1}$ and $\sigma_{1}$, and the values of $x_{0}$ and a suitable $\Delta t$.

(b) Calculate $\phi_{0}$ and $A_{0}$ using eqn 12. Let $t=\Delta t, n=0$.

(c) Calculate $A_{n+1}=A_{n} \exp \left\{\sigma_{1}\left(A_{n}\right)\right\} \Delta t ; \phi_{n+1}=\phi_{n}+\omega_{1}\left(A_{n}\right) \Delta t$ and $x_{n+1}=A_{n+1} \sin \left(\phi_{n+1}\right)$. Plot $x_{n+1}, A_{n+1}, t$. Let $t=t+\Delta t$ and let $n=n+1$. Calculate $\sigma_{1}\left(A_{n}\right), \omega_{1}\left(A_{n}\right)$ from the input data, $A_{n}$ and eqn. 10 , where $\dot{\bar{\omega}}=\left\{\bar{\omega}\left(A_{n}\right)\right.$ $\left.-\bar{\omega}\left(A_{n-1}\right)\right\} / \Delta t$ and $\dot{\bar{\sigma}}=\left\{\bar{\sigma}\left(A_{n}\right)-\bar{\sigma}\left(A_{n-1}\right)\right\} / \Delta t$. (d) Repeat step (c) until a sufficient portion of the transient oscillations are calculated.

Example: For the system equation

$$
\left[s^{4}+36 s^{3}+335 s^{2}+\left\{300+15 K N_{1}(A)\right\} s+300 K\right] x=0
$$

the $\alpha \beta$ parameter-plane diagram is in Fig. 1, where $\alpha=K$ and $\beta==K N_{1}(A)$. The design problem is to select $K$ and $N_{1}(A)$ so that the transient oscillations have less than $15 \%$ overshoot for initial values of $x$ from $x=0$ to $x=10$, and the 'fall' time and settling time are to be as short as possible. This quasioptimisation problem has been solved in the parameter plane using a value of 525 for $K$ and choosing $N_{1}(A)$ corresponding to a saturating element (see the $M$ locus of Fig. 1). The solution $x(t)$ of eqn. 13 may be calculated approximately using the parameter-plane diagram information and the algorithm above. This has been done for three sets of initial conditions and the results are plotted in Fig. 2. Various responses of a second-order linear system are also given in Fig. 2 for comparison. The exact response of eqn. 13 for the case of Fig. 1 is given, to within a few percent, by the approximate responses of Fig. 2. Other cases have been calculated with a similar accuracy, including one in which two nonlinearities were involved. Extensions to the case when the $M$ locus enters the part of the parameter plane for which the dominant roots are real are readily made.

We comment that, for the second-order nonlinear system (eqn. 5), it is possible to determine the response from the location of the roots and the time derivative of the location of the roots to a high degree of approximation, if the calculations of $\phi_{0}$ and $A_{0}$ (eqn. 12) are sufficiently good. This result, together with the parameter-plane method, forms the basis of useful approximate design procedures for higherorder systems.

J. B. MOORE

10th October 1967

Department of Electrical Engineering

University of Newcastle

Newcastle, NSW, Australia

\section{References}

1 SIJAK, D. D.: 'Analysis and synthesis of feedback control systems in the parameter plane', IEEE Trans., Power Apparatus Syst., 1964, 83, pp. $466-473$

2 SILJAK, D. D.: 'Generalisation of the parameter plane method', IEEE Trans, 1966, AC-11, pp. 63-70

3 MOORE "Control system design using extensions of the parameter plane concept', Ph.D. thesis, University of Santa Clara, California, 1967

4 GRENSTED, P. E. W.: 'Analysis of the transient response of nonlinear control systems', Trans $A S M E, 1958,80$, pp. $247-253$ 